\title{
Unusual Case of Pulmonary and Hepatic Metastases of Mandibular Ameloblastoma
}

\author{
NALINI KILARA, MURALI SUBRAMANIAN AND KIRTHI KOUSHIK
}

\begin{abstract}
:
Ameloblastoma, a tumour of odontogenic origin constitutes $1 \%$ of all tumours of jaw and $11 \%$ of all odontogenic tumours. Fewer than 45 cases of Ameloblastoma with metastasis have been reported till date. Here we report a case of Ameloblastoma of the mandible with hepatic and pulmonary metastasis about 14 years post surgical resection of the primary tumour with the primary disease under control.
\end{abstract}

\section{INTRODUCTION}

Ameloblastomas are benign, locally invasive tumours diagnosed most commonly in third to fifth decade of life. Gender distribution varies but is probably about 1:1 and most authors report an equal racial distribution. Patients commonly present with painless swelling of jaw and additional symptoms can include malocclusion, pain, tooth mobility, ill fitting dentures, periodontal disease, ulceration, paresthesia and/or anesthesia of the affected area.

Histologically Ameloblastomas arise from cell remnants of embryonic tooth mainly the dental lamina or inner enamel epithelium. They are composed of central area of stellate epithelial cells known as the stellate reticulum and a periphery of vacuolated columnar cells known as reserve cells. Ameloblastomas are of 2 common histological patterns: follicular and plexiform. Other less common variants can include acanthomatous, granular, basaloid and

Departments of Medical Oncology, M S Ramaiah Medical College, Bangalore - 560054, India

Correspondence to: NALINI KILARA

Email: nalini_kilara@yahoo.com desmoplastic. These tumours are subdivided based on overall histological architecture into 4 variants-solid, multicystic, unicystic and combination.

Two forms of rare malignancy are associated with Ameloblastoma: malignant Ameloblastoma and Ameloblastic carcinoma. The cardinal feature of malignant Ameloblastoma is metastatic spread and the histological appearance of primary and metastatic lesions are indistinguishable from benign Ameloblastoma. In contrast, Ameloblastic carcinoma shows histologically malignant epithelial features similar to epidermoid carcinoma.

CASE : A 39-year-old female patient presented to us with complaints of vomiting since two and half months. She gave history of local excision of the mandible for Ameloblastoma in 1993, which recurred in 1995, and she had undergone reexcision with PMMC and Silastic mandible. She remained disease free till September 2006 when she developed above symptoms.

On abdominal examination a mass was palpable in the epigastric and right hypochondriac regions and ultrasound abdomen showed a hypoechoic lesion in both lobes of liver. CT abdomen showed a 9x9x8.6cms mass in the left lobe of liver with heterogeneous enhancement. The chest X-ray revealed a coin shaped lobulated soft tissue opacity in the left mid zone. Chest CT scan showed a soft tissue mass measuring $7 \times 5.3 \times 4.2 \mathrm{cms}$ with loss of plane between mass and pleura and pericardium.

Biopsy of the liver mass showed islands of basaloid epithelial cells, focal squamoid metaplasia and cells with round to oval nuclei consistent with Ameloblastoma. Rest of the work up was within normal limits. 


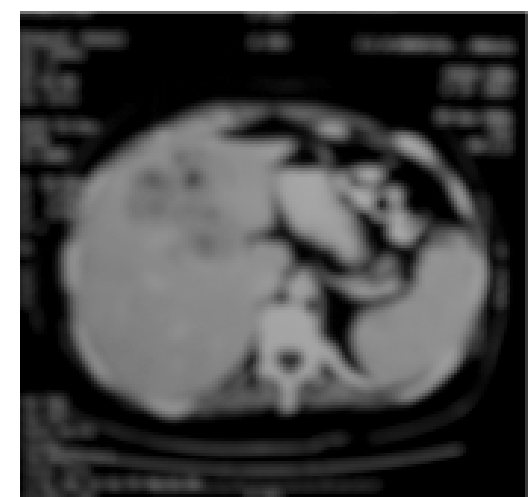

Fig 1: CT abdomen showed a 9x9x8.6cms mass in the left lobe of liver with heterogeneous enhancement.

She was treated symptomatically and was advised about the different treatment modalities available along with the outcome of each of them. She became symptomatically better but declined to undergo any kind of tumour related therapy. Patient expired a few months later.

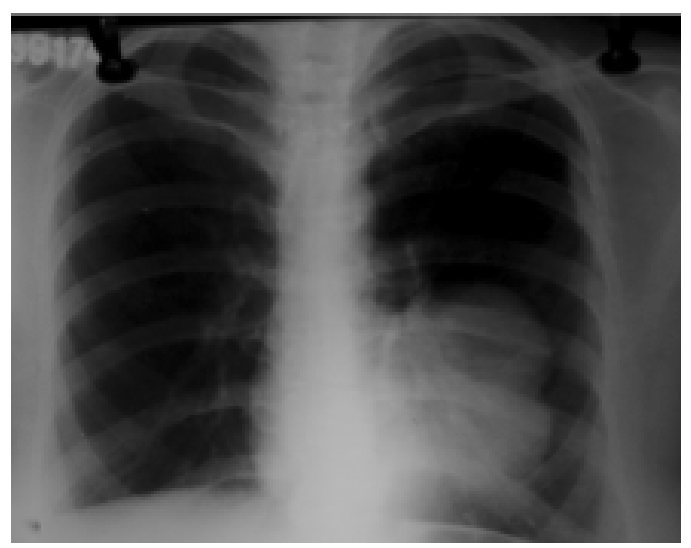

Fig 2: The chest X-ray revealed a coin shaped lobulated soft tissue opacity in the left mid zone.

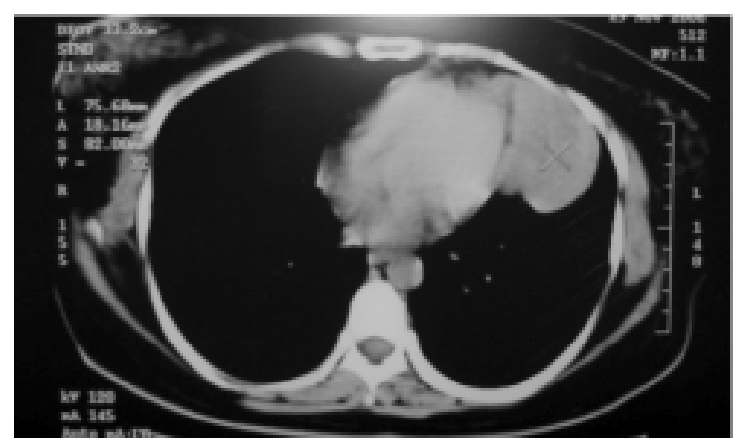

Fig 3: Chest CT scan showed a soft tissue mass measuring $7 \times 5.3 \times 4.2 \mathrm{cms}$ with loss of plane between mass and pleura and pericardium.

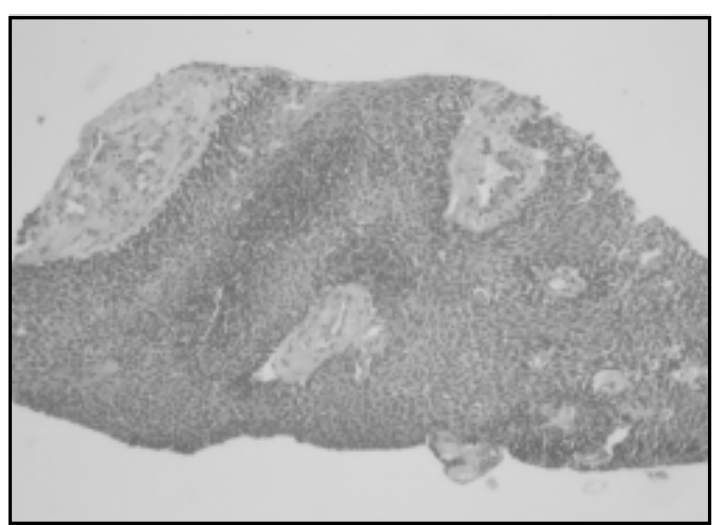

Fig 4: Biopsy of the liver mass showed islands of basaloid epithelial cells, focal squamoid metaplasia and cells with round to oval nuclei consistent with Ameloblastoma.

\section{DISCUSSION}

Ameloblastoma is a rare tumour usually occurring in the jaw particularly mandible and rarely metastasizes. Less than 45 cases of Ameloblastoma with metastasis have been reported in literature This tumour is known to have a high local recurrence rate (50-72\%).

The most frequent site of metastasis is lung, which is seen in up to $85 \%$ of the cases followed by lymph node, pleura, liver and parotid gland. Some investigators believe that pulmonary capillaries are an effective barrier to further dissemination as only a limited number of patients with extrapulmonic metastatic disease can be identified. In the present case there was presence of both liver and lung metastasis. The chances of metastasis increase with the duration from the time of presentation of primary and also the number of local recurrences. The common routes of spread are lymphatic and haematogenous but Vorzimer and Perla have put forward the concept of aspirated neoplastic cells as the cause of pulmonary metastases.

The various treatment options available are: Surgery, Radiotherapy and Chemotherapy. Surgery is the optimal treatment for patients with Ameloblastoma and Ameloblastic carcinoma. The optimal surgical approach is also controversial with options ranging from enucleation, curettage, cryotherapy, electrocautery, marsupialization and finally 
wide resection with a margin of $>1 \mathrm{~cm}$ of uninvolved cancellous bone is accepted by many surgeons. Gardner and Pecak reported that conservative approaches are suitable only for unicystic lesions and if the tumour is situated in the posterior part of maxilla then aggressive management is necessary due to proximity to vital structures and difficulty in managing the recurrences. ${ }^{6}$ Due to the high risk of local recurrences and low rate of control with salvage therapy adjuvant therapy should be considered especially in high risk individuals.

There is limited data pertaining to efficacy of radiotherapy (RT). Based on the results of Gardner, RT can produce regression of an Ameloblastoma, particularly the part which causes expansion of the jaw or has invaded the adjacent soft tissues but that it is appropriate treatment only for unresectable tumours? ${ }^{7}$.

Data pertaining to chemotherapy is sparse and only anecdotal reports are available. Lanham described a case report of Ameloblastoma metastatic to lungs and nodes treated with doxorubicin, cisplatin, Cyclophosphamide, dacarbazine and 5FU but the tumour failed to respond. ${ }^{3}$ Grunwald et al has shown execellent response to paclitaxel and carboplatin combination. ${ }^{8}$

There are no clear-cut guidelines regarding treatment of metastases except for a few anecdotal reports which advise the role of frequent follow-up after the appearance of recurrence of primary. Neither chemotherapy nor radiotherapy have shown significant benefit. Surgery in selected cases which are amenable for resection has shown a survival benefit. The mean time from appearance of metastasis to death is around 16 months.

Owing to the rarity of the tumours and the short survival after recurrence further research is necessary to accurately say which modality of therapy is most effective.

\section{REFERENCES:}

1. Mehlisch DR, Dahlin DC, Masson JK. Ameloblastoma:clinicopathologic report." J.Oral Surg 1972;30:9-22.

2. Lawrence M. Climent and Ari J. Climent, "Malignant Ameloblastoma metastatic to lungs 29 years after primary resection: a case report." Chest, 2002;121:1359-1361.

3. Lanham $R$." Chemotherapy of metastatic Ameloblastoma: a case report and review of literature." Oncology, 1987;44:133-134.

4. Donath $K$, Kunze $E$ and Luhr $H$ G, "Biology of Metastasizing Ameloblastoma." Pathol res pract: 1985;180;526-535.

5. Vorzimer J Perla D. An instance of Ameloblastoma of jaw with metastases to right lung" $\mathrm{Am} j$. Pathology, 1932;8:445-453.

6. Gardner DG, Pecak AM."The treatment of ameloblastoma based on pathologic and anatomic principles." Cancer, 1980;46:2514-2519.

7. Gardner DG. Radiotherapy in the treatment of ameloblastoma." Int $J$ Oral Maxillofac Surg: 1988;17:201-205.

8. Grunwald V,Leblanc $S$, Karstens $J H$ et al."metastatic malignant ameloblastoma responding to chemotherapy with Paclitaxel and Carboplatin." Ann Oncol 2001;12:1489-1491.

9. Sheppard B C, Temeck B K, Taubenberger and Pass $H$ I, "Pulmonary metastatic disease in Ameloblastoma." Chest: 1993;104:1933-1935. 